3. Наиболее полную опорную кривую имеет поверхность втулки, обработанная раскатыванием; которая при $\varepsilon \leq 0,3$ составляет 0,65 , а ее износостойкость значительно выше чем хонингованных абразивными и алмазными брусками.

\title{
Библиографические список:
}

1. Исследование температуры поршня, гильзы, изменения температуры газа при сгорании топлива в вихрекамерном двигателе 4ч 8,5/11. Отчет. Харьковский институт инженеров железнодорожного транспорта. Чиркин А.П., Симсон А.Э и др. 1965, 70 с. 2. Крагельский И.В., М.Н., Комбалов В.С. Основы расчетов на трение и износ. М.; 1977.

3. Санаев Н.К., Тынянский В.П. Исследование износа и метода ускоренных испытаний предварительно упрочненных поршневых канавок ДВС. Вестник Дагестанского технического университета. 2012.

4. Махин Н.М. О связи площади касания и сближения при неподвижном и скользящем контакте. Сб. «Трение и износ тел.» М.; Наука, 1971.

5. Крагельский И.В и др. Основы расчетов на трение и износ. М.; Машиностроение, 1977, С. 525.

6. Дизели - методы испытаний на надежность. ОСТ 24.060.08., М.; 1975.

УДК. 656.13

Агаханов Э.К., Батманов Э.З.

ОПРЕДЕЛЕНИЕ НАЧАЛЬНЫХ УСЛОВИЙ ДОРОЖНО-ТРАНСПОРТНЫХ ПРОИСШЕСТВИЙ ПО ИХ ПОСЛЕДСТВИЯМ

Agakhanov E.K., Batmanov E.Z.

\section{DETERMINATION OF THE INITIAL CONDITIONS OF ROAD TRAFFIC ACCIDENTS IN THEIR CONSEQUENCES}

Разработана расчетно-экспериментальная методика определения начальных условий дорожно-транспортных происшествий по их последствиям при фронтальном столкновении.

Ключевые слова: дорожно-транспортное прочсшествие; начальные условия; последствия; фронтальное столкновение.

Developed a calculation-experimental method of determining the initial conditions of road traffic accidents in their consequences in the event of a frontal collision.

Key words: road traffic accident; initial conditions; effects; frontal collision.

Существенным недостатком статистического метода оценки пассивной безопасности автотранспортных средств является отсутствие полных данных о начальных условиях дорожно-транспортных происшествий, в частности, сведений о скорости автомобиля в момент происшествия. Без таких данных трудно планировать и осуществлять мероприятия по повышению безопасности конструкции автотранспортных средств, только при известных параметрах нормативных (эталонных) испытаний возможно проводить доводку конструкции. Поэтому, получив из анализа последствий дорожно-транспортных происшествий данные по деформациям (перемещениям) отдельных элементов 
автомобиля, прежде чем приступать к разработке мероприятий по повышению безопасности, необходимо определить начальные условия дорожно-транспортных происшествий.

В настоящее время для этого могут быть использованы два способа:

- экспериментальный способ с проведением большого объема испытаний для эмпирического получения тех значений деформаций, которые были зафиксированы в условиях дорожно-транспортных происшествий;

- расчетно-экспериментальный способ с использованием расчетных моделей заранее построенных (проверенных) по результатам ограниченного числа экспериментов.

Понятно, что первый способ весьма дорогостоящий и может быть использован в весьма ограниченных масштабах. В частности, таким способом американские исследователи получили данные, использованные в дальнейшем для установления нормативов по ударно-прочностным характеристикам верхней части кузова (крыши) легкового автомобиля.

Наиболее приемлемым является второй способ.

Более достоверными и стабильными показателями, которые возможно с достаточной точностью ( \pm 5 мм) замерить на поврежденном в результате фронтального столкновения автомобиле при дорожно-транспортных происшествиях, являются величины остаточных деформаций автомобиля и перемещения панели приборов вглубь салона.

Для скоростей наезда, близких к 50 км/час, может быть использована описанная ниже математическая модель фронтального столкновения легкового автомобиля с жестким неподвижным препятствием.

Представим автомобиль в виде недеформируемой массы, а совокупность всех сил, действующих в процессе фронтального столкновения заменим условным упругим элементом с постоянным коэффициентом жесткости $\mathrm{C}_{1}$; все силы, характеризующие необратимые потери энергии представим в виде гидравлического амортизатора (вязкое трение) с коэффициентом сопротивления $\mu_{1}$. Остальные силы, действующие на автомобиль в процессе наезда, (как это принято в теории удара) не учитываем.Форма дифференциального управления движения автомобиля в процессе деформации передней части зависит от начальной скорости. Так, при больших начальных скоростях $\left(\mathrm{V}_{0}>80 \ldots 100 \mathrm{\kappa} /\right.$ ч) сопротивление гидроамортизатора можно принять пропорциональным квадрату скорости. При этом уравнение движения может быть написано в следующем виде:

$$
\mathrm{m} \ddot{\mathrm{X}}+\mu \dot{\mathrm{X}}^{2}+\mathrm{C}_{1} \mathrm{X}=0
$$

где $\mathrm{m}$ - полная масса автомобиля, кг;

$\mathrm{C}_{1}$ - приведенная жесткость передней части автомобиля, Н/м;

$\mu_{1}$ - приведенный коэффициент демпфирования передней части, Нc/м;

$\mathrm{X}$ - перемещение центра тяжести, м.

Точки над символами означают производные координаты по времени, а индексы номер фазы.

Уравнение (1) является нелинейным уравнением второго порядка, не имеющим аналитического решения. Покажем, как оно может быт решено при использовании метода приравнивания степеней. Для этого перепишем его следующим образом:

$$
\ddot{\mathrm{X}}+\frac{\mu_{1}}{\mathrm{~m}} \dot{\mathrm{X}}^{2}+\frac{\mathrm{C}_{1}}{\mathrm{~m}} \mathrm{X}=0 .
$$

Решение уравнения ищем в виде полинома n-ной степени:

$$
\mathrm{X}=\mathrm{a}_{0}+\mathrm{a}_{1} \mathrm{t}+\mathrm{a}_{2} \mathrm{t}^{2}+\mathrm{a}_{3} \mathrm{t}^{3}+\ldots+\mathrm{a}_{\mathrm{n}} \mathrm{t}^{\mathrm{n}},
$$

где $\quad a_{0} \ldots a_{n}$ - эмпирические коэффициенты;

t- время, с.

Продифференцируем полином (3) по времени: 


$$
\begin{aligned}
& \dot{X}=a_{1}+2 a_{2} t+3 a_{3} t^{2}+\ldots+n a_{n} t^{n-1} ; \\
& \ddot{X}=2 a_{2}+6 a_{3} t+\ldots+n(n-1) a_{n} t^{n-2} .
\end{aligned}
$$

Учитывая кратковременность процесса (продолжительность первой стадии не превышает 0,1 с), в связи с чем члены, содержащие высокие степени тстановятся пренебрежимо малыми, ограничимся треть степенью аргумента:

$$
\begin{gathered}
X=a_{0}+a_{1} t+a_{2} t^{2}+a_{3} t^{3} ; \\
\dot{X}=a_{1}+2 a_{2} t+3 a_{3} t^{2}+4 a_{4} t^{3} ; \\
\ddot{X}=2 a_{2}+6 a_{3} t+12 a_{4} t^{2}+20 a_{5} t^{3} .
\end{gathered}
$$

Тогда

$$
\begin{aligned}
& \dot{X}^{2}=a_{1}{ }^{2}+4 a_{2}{ }^{2} t^{2}+9 a_{3}{ }^{2} t^{4}+16 a_{4} t^{6}+4 a_{1} a_{2} t+ \\
& +6 a_{1} a_{3} t^{2}+8 a_{1} a_{4} t^{3}+12 a_{2} a_{3} t+16 a_{2} a_{4} t^{4}+24 a_{3} a_{4} t^{5}
\end{aligned}
$$

Подставим значения $\mathrm{X}, \dot{\mathrm{X}}$ и $\ddot{\mathrm{X}}$ в уравнение (2) в соответствии с формулами (6), (8) и (9):

$$
\begin{aligned}
& 2 \mathrm{a}_{2}+6 \mathrm{a}_{3} \mathrm{t}+12 \mathrm{a}_{4} \mathrm{t}^{2}+20 \mathrm{a}_{5} \mathrm{t}^{3}+ \\
& +\left(\mathrm{a}_{1}{ }^{2}+4 \mathrm{a}_{2}{ }^{2} \mathrm{t}^{2}+9 \mathrm{a}_{3}{ }^{2} \mathrm{t}^{4}+16 \mathrm{a}_{4} \mathrm{t}^{6}+4 \mathrm{a}_{1} \mathrm{a}_{2} \mathrm{t}+6 \mathrm{a}_{1} \mathrm{a}_{3} \mathrm{t}^{2}++8 \mathrm{a}_{1} \mathrm{a}_{4} \mathrm{t}^{3}+12 \mathrm{a}_{2} \mathrm{a}_{3} \mathrm{t}^{3}+16 \mathrm{a}_{2} \mathrm{a}_{4} \mathrm{t}^{4}+24 \mathrm{a}_{3} \mathrm{a}_{4} \mathrm{t}^{5}\right) \\
& \cdot \frac{\mu_{1}}{\mathrm{~m}}+\left(\mathrm{a}_{0}+\mathrm{a}_{1} \mathrm{t}+\mathrm{a}_{2} \mathrm{t}^{2}+\mathrm{a}_{3} \mathrm{t}^{3}\right) \cdot \frac{\mathrm{C}_{1}}{\mathrm{~m}}=0
\end{aligned}
$$

Сгруппируем члены с одинаковыми степенями t:

$$
\begin{aligned}
& \left(9 \mathrm{a}_{3}{ }^{2}+16 \mathrm{a}_{2} \mathrm{a}_{4}\right) \frac{\mu_{1}}{\mathrm{~m}} \mathrm{t}^{4}+\left[20 \mathrm{a}_{5}+\left(8 \mathrm{a}_{1} \mathrm{a}_{4}+12 \mathrm{a}_{2} \mathrm{a}_{3}\right) \frac{\mu_{1}}{\mathrm{~m}}+\mathrm{a}_{3} \frac{\mathrm{C}_{1}}{\mathrm{~m}}\right] \mathrm{t}^{3}+ \\
& +\left[12 \mathrm{a}_{4}+\left(4 \mathrm{a}_{2}^{2}+6 \mathrm{a}_{3} \mathrm{a}_{1}\right) \frac{\mu_{1}}{\mathrm{~m}}+\mathrm{a}_{2} \frac{\mathrm{C}_{1}}{\mathrm{~m}}\right] \mathrm{t}^{2}+\left(6 \mathrm{a}_{3}+4 \mathrm{a}_{1} \mathrm{a}_{2} \frac{\mu_{1}}{\mathrm{~m}}+\mathrm{a}_{1} \frac{\mathrm{C}_{1}}{\mathrm{~m}}\right) \mathrm{t}+ \\
& +\left(2 \mathrm{a}_{2}+\mathrm{a}_{1}{ }^{2} \frac{\mu_{1}}{\mathrm{~m}}+\mathrm{a}_{0} \frac{\mathrm{C}_{1}}{\mathrm{~m}}\right)=0
\end{aligned}
$$

Начальные условия движения: в момент времени $\mathrm{t}=0 ; \mathrm{X}(0)=0 ; \dot{\mathrm{X}}(0)=\mathrm{V}_{0}$, где $\mathrm{V}_{0}-$ начальная скорость автомобиля (скорость в момент контакта передней части автомобиля с препятствиями). В соответствии с этими имеем:

$$
\begin{gathered}
\text { при } \mathrm{t}^{0} 2 \mathrm{a}_{2}+\mathrm{a}_{1}{ }^{2} \frac{\mu_{1}}{\mathrm{~m}}+\mathrm{a}_{0} \frac{\mathrm{C}_{1}}{\mathrm{~m}}=0, \\
\text { при } \mathrm{t}^{1} 6 \mathrm{a}_{3}+4 \mathrm{a}_{1} \mathrm{a}_{2} \frac{\mu_{1}}{\mathrm{~m}}+\mathrm{a}_{1} \frac{\mathrm{C}_{1}}{\mathrm{~m}}=0, \\
\text { при } \mathrm{t}^{2} 12 \mathrm{a}_{4}+\left(4 \mathrm{a}_{2}{ }^{2}+6 \mathrm{a}_{1} \mathrm{a}_{3}\right) \frac{\mu_{1}}{\mathrm{~m}}+\mathrm{a}_{2} \frac{\mathrm{C}_{1}}{\mathrm{~m}}=0, \\
\text { при } \mathrm{t}^{3} 20 \mathrm{a}_{5}+\left(8 \mathrm{a}_{1} \mathrm{a}_{4}+12 \mathrm{a}_{2} \mathrm{a}_{3}\right) \frac{\mu_{1}}{\mathrm{~m}}+\mathrm{a}_{3} \frac{\mathrm{C}_{1}}{\mathrm{~m}}=0,
\end{gathered}
$$

и т.Д.

Из уравнения (6) при указанных начальных условиях получаем:

$$
\mathrm{X}(0)=\mathrm{a}_{0}=0
$$

Тогда из (11)

$$
2 \mathrm{a}_{2}=-\mathrm{a}_{1}^{2} \frac{\mu_{1}}{\mathrm{~m}}
$$


Соответственно из выражения (7) при $\mathrm{t}=0$

Из (16) и (17)

$$
\dot{\mathrm{X}}(0)=\mathrm{a}_{1}=\mathrm{V}_{0} \text {. }
$$

$$
\mathrm{a}_{2}=-\frac{\mathrm{V}_{0}^{2}}{2} \cdot \frac{\mu_{1}}{\mathrm{~m}}
$$

Поскольку коэффициенты а 1 и а2 определены, то из уравнения (12) можно найти значение коэффициента аз:

$$
\mathrm{a}_{3}=-\frac{1}{6}\left(4 \mathrm{a}_{1} \mathrm{a}_{2} \frac{\mu_{1}}{\mathrm{~m}}+\mathrm{a}_{1} \frac{\mathrm{C}_{1}}{\mathrm{~m}}\right)=-\frac{1}{6}\left(-4 \mathrm{~V}_{0} \frac{\mathrm{V}_{0}^{2}}{2} \frac{\mu_{1}}{\mathrm{~m}}+\mathrm{V}_{0} \mathrm{C}_{1}\right)=\frac{\mathrm{V}_{0}}{6 \mathrm{~m}}\left(2 \mathrm{~V}_{0}{ }^{2} \frac{\mu_{1}^{2}}{\mathrm{~m}}-\mathrm{C}_{1}\right) .
$$

Продолжая таким же образом из уравнений (13) и (15) можно найти значения остальных коэффициентов а4, a5,.., $\mathrm{a}_{\mathrm{n}}$.

Следовательно, при известных характеристиках системы $\left(\mathrm{m} ; \mu_{1} ; \mathrm{C}_{1}\right)$ уравнение $(2)$ можно считать решенным. Уравнение (3) после подстановки численных значений эмпирических коэффициентов $\mathrm{a}_{0}, \ldots, \mathrm{a}_{\mathrm{n}}$, позволяет определить положение центра тяжести в различные моменты времени. Напротив, если из эксперимента известны функциональные зависимости некоторых параметров (например, $\mathrm{X}=\mathrm{X}(\mathrm{t})$ или $\dot{\mathrm{X}}=\dot{\mathrm{X}}(\mathrm{X})$ и т.д.), можно найти значения коэффициентов $\mathrm{C}_{1}$ и $\mu_{1}$.

При относительно невысоких скоростях (порядка 40-50км/ч) гидравлическое сопротивление можно считать пропорциональным первой степени $\dot{\mathrm{X}}$. В этом случае уравнение (1) становится линейным и приобретает вид:

$$
\mathrm{m} \dot{\mathrm{X}}+\mu_{1} \dot{\mathrm{X}}+\mathrm{C}_{1} \mathrm{X}=0 \text {. }
$$

Для решения его обычно представляют в следующей форме:

$$
\ddot{X}+2 n_{1} \dot{X}+\omega_{1}^{2} X=0 \text {, }
$$

Где

$$
\mathrm{n}_{1}=\frac{\mu_{1}}{2 \mathrm{~m}} ; \quad \omega_{1}=\sqrt{\frac{\mathrm{C}_{1}}{\mathrm{~m}}} .
$$

Уравнение (21) имеет аналитическое решение:

где

$$
\begin{gathered}
X=\mathrm{e}^{-\mathrm{n}_{1} \mathrm{t}}\left[\mathrm{C}_{1} \cos \left(\omega_{1} \mathrm{t}^{\mathrm{t}}\right)+\mathrm{C}_{2} \sin \left(\omega_{1} \mathrm{t}\right)\right], \\
\omega_{1}{ }^{\prime}=\sqrt{\omega_{1}{ }^{2}-\mathrm{n}_{1}{ }^{2}} ;
\end{gathered}
$$

условий.

$\mathrm{C}_{1}, \mathrm{C}_{2}$ - постоянные интегрирования, которые определяют из начальных

Для определения постоянных дифференцируем (22) по времени:

$$
\dot{X}=e^{-n_{1} t}\left[\cos \omega_{1}^{\prime} t \cdot\left(n_{1} C_{1}+\omega_{1} C_{2}\right)+\sin \omega_{1} t\left(-\omega_{1} C_{1}-n_{1} C_{2}\right)\right]
$$

Используя те же начальные условия, что и выше, получаем:

$$
\mathrm{C}_{1}=0 ; \quad \mathrm{C}_{2}=\frac{\mathrm{V}_{0}}{\omega_{1}^{\prime}} ;
$$

Тогда

$$
\begin{gathered}
\mathrm{X}=\frac{\mathrm{V}_{0}}{\omega_{1}^{\prime}} \cdot \mathrm{e}^{-\mathrm{n}_{1} \mathrm{t}} \sin \omega_{1} \mathrm{t}^{\mathrm{t}} \\
\dot{\mathrm{X}}=\mathrm{V}_{0} \mathrm{e}^{-\mathrm{n}_{\mathrm{t}} \mathrm{t}}\left(\cos \omega_{1} \mathrm{t}-\frac{\mathrm{n}_{1}}{\omega_{1}^{\prime}} \sin \omega_{1} \mathrm{t}^{\mathrm{t}}\right) .
\end{gathered}
$$

Последние формулы дают возможность при известных характеристиках системы определить перемещение и скорость центра тяжести в момент времени $\mathrm{t}$.

Так, например, можно определить продолжительность первой фазы удара и перемещение центра тяжести с начала контакта его с препятствием до остановки, т.е. 
максимальную деформацию передней части. В момент остановки (конец первой фазы удара) $\Delta \max$ и $\dot{\mathrm{X}}=0$, поэтому из (24) получаем:

$$
\operatorname{tg}\left(\omega_{1}^{\prime} \tau_{1}\right)=\frac{\omega_{1}^{\prime}}{n_{1}},
$$

где $\tau_{1-}$ продолжительность первой фазы, с.

Следовательно, время:

$$
\tau_{1}=\frac{1}{\omega_{1}^{\prime}} \operatorname{arctg}\left(\frac{\omega_{1}^{\prime}}{n_{1}}\right) .
$$

Максимальная деформация $\Delta \max$ согласно (23) и (26) составляет:

$$
\Delta \max =\frac{\mathrm{V}_{0}}{\omega_{1}^{\prime}} \mathrm{e}^{-\mathrm{n}_{1} \tau_{1}} \sin \omega_{1}^{\prime} \tau_{1} .
$$

Приведенная выше модель удара позволяет решить как прямую задачу динамики определение параметров движения тел по известным силам, так и обратную задачу нахождение характеристики системы по известному закону движения.

Покажем, как с помощью разработанной модели можно определить значения характеристик $\mathrm{C}$ и $\mu$ упругого и вязкого сопротивлений. Поскольку в процессе удара свойства системы меняются и элементы автомобиля, деформированные в течение первой фазы, хотя затем и частично восстанавливают свою первоначальную форму, то величины $\mathrm{C}_{1}$ и $\mathrm{C}_{2}$ не совпадают. Поэтому их определяем раздельно для каждой из фаз удара.

Рассмотрим первую фазу наезда. Определим из уравнения (25) $\mathrm{n}_{1}$ :

$$
\mathrm{n}_{1}=\frac{\omega_{1}^{\prime}}{\operatorname{tg}\left(\omega_{1}^{\prime} \tau_{1}\right)} \text {. }
$$

Подставим его значение $\mathrm{n}_{1}$ в уравнение (23):

$$
\frac{\Delta_{1} \omega_{1}{ }^{\prime}}{V_{1}}=\mathrm{e}^{\frac{\omega_{1}^{\prime} \tau_{1}}{\operatorname{tg}\left(\omega_{1}^{\prime} \tau_{1}\right)}}=\sin \omega_{1}{ }^{\prime} \tau_{1} .
$$

Для краткости записи обозначим: $\omega_{1} \tau_{1}=\mathrm{X}_{1}$, тогда

$$
\frac{\Delta_{1} \lambda_{1}}{V_{1} \tau_{1}} \mathrm{e}^{\frac{\lambda_{1}}{\operatorname{tg} \lambda_{1}}}=\sin \lambda_{1}
$$

Это трансцендентное уравнение содержит лишь одно неизвестное - $\lambda_{1}$. Остальные величины $\left(\mathrm{V}_{1}, \Delta_{1}, \tau_{1}\right)$ определяем по графику «время-перемещение». Решая уравнение (30) графически, методом итерации или каким-либо другим путем находим $\lambda_{1}$. Далее определяем коэффициент вязкого сопротивления в первой фазе наезда:

$$
\mu_{1}=2 \mathrm{mn}_{1}=2 \mathrm{~m} \frac{\lambda}{\tau_{1} \operatorname{tg} \lambda_{1}} .
$$

Коэффициент жесткости в первой фазе:

$$
\mathrm{k}_{1}=\mathrm{m}_{1} \frac{\lambda_{1}{ }^{2}}{\tau_{1}{ }^{2} \sin ^{2} \lambda_{1}} .
$$

Используя данную модель возможно расчетным путем определять значение скорости автомобиля при наезде на неподвижное препятствие по значению максимальной (остаточной) деформации. Эту же модель, возможно, использовать для определения предельного уровня пассивной безопасности автомобиля.

Приведенная выше модель позволяет определить деформацию X и скорость $\dot{\mathrm{X}}$ автомобиля как функции времени: 


$$
\begin{gathered}
\mathrm{X}=\mathrm{X}(\mathrm{t}) \\
\dot{\mathrm{X}}=\dot{\mathrm{X}}(\mathrm{t}) .
\end{gathered}
$$

Как X, так и $\dot{\mathrm{X}}$ непосредственно зависит от начальной скорости автомобиля перед ударом:

$$
\begin{gathered}
\mathrm{X}=\mathrm{X}_{1}\left(\mathrm{~V}_{0}\right) \\
\dot{\mathrm{X}}=\dot{\mathrm{X}}_{1}\left(\mathrm{~V}_{0}\right) .
\end{gathered}
$$

Варьируя значения $\mathrm{V}_{0}$,можно получить зависимости перемещения и скорости от $\mathrm{V}_{0}$ через определенные промежутки времени t. В свою очередь, перемещения деталей интерьера автомобиля, ограничивающих жизненное пространство, связаны с деформацией передней части. Аналитически эту связь установить довольно трудно, однако экспериментальное определение сложности не представляет. При правильно проведенных сериях экспериментов можно получить эмпирическое выражение:

$$
\delta_{\mathrm{i}}=\Delta_{1}(\mathrm{X})
$$

где $\delta_{\mathrm{i}}$ - перемещение детали интерьера (рулевое колесо, рулевая колонка, панель прибора и т.д.).

В результате для каждой модели автомобиля может быть установлена зависимость:

$$
\delta_{\mathrm{i}}=\delta\left(\mathrm{V}_{0}\right)
$$

характеризующая перемещение рассматриваемой травмоопасной детали интерьера (в частности, панели приборов) от начальной скорости автомобиля перед его наездом на препятствие.

К сожалению, имеющиеся экспериментальные данные характеризуют перемещение деталей лишь в узком диапазоне скоростей, что не дает возможности их использования для более глубокого анализа исследуемого процесса в большом диапазоне.Покажем, как это можно сделать на основании проведенного выше аналитического исследования наезда автомобиля на жесткое препятствие.Рассмотрим следующую формулу:

$$
\Delta_{\max }=\frac{\mathrm{V}_{0}}{\omega_{1}^{\prime}} \cdot \mathrm{e}^{-\mathrm{n}_{1} \tau_{1}} \sin \left(\omega_{1}{ }^{\prime} \tau_{1}\right) .
$$

В этой формуле параметры $\mathrm{n}_{1}$ и $\omega_{1}^{\prime}$, обусловлены конструкцией автомобиля. Они характеризуют потери энергии на преодоление внутреннего трения $\left(\mathrm{n}_{1}\right)$ и упругие деформации деталей Со значительной степенью вероятности их можно считать постоянными для данного конструктивного решения в ограниченном диапазоне скорости наезда.

Продолжительность первой фазы удара (параметр $\tau_{1}$ ), вообще говоря, зависит от начальной скорости $\mathrm{V}_{0}$, увеличиваясь с ростом последней. Однако, в нашем случае это не имеет существенного значения и его в первом приближении, по-видимому, также можно принять за постоянную величину. Основание к этому следующее.

Во-первых, как показали испытания одной и той же модели автомобиля при разных начальных скоростях $(31,40,50,60 \kappa \mathrm{q} /$ п) продолжительность первого периода удара практически не меняется. Растет лишь конечная деформация передней части автомобиля.

Во-вторых, функция $\mathrm{e}^{-\mathrm{n}_{1} \tau_{1}}$ уменьшается с увеличением показателя, т.е. с ростом $\tau_{1}$. Функция $\sin \left(\omega_{1}^{\prime} \tau_{1}\right)$, напротив, увеличивается. Учитывая, что практически $\tau_{1}$ может возрастать не на порядок, а всего на несколько процентов, изменение указанных функций должно взаимно компенсировать друг друга. В результате произведение этих функций, а, следовательно, все члены при $\mathrm{V}_{0}$ правой части формулы могут считаться постоянными.

Покажем справедливость принятого допущения на примере.

Обработка результатов испытания автомобиля ВАЗ-2108 (масса 945кг) дала следующие значения характеристик системы: 


$$
\mathrm{K}_{1}=316000 \mathrm{H} / \mathrm{M} ; \quad \mu_{1}=12400 \mathrm{H} \cdot \mathrm{M} \cdot \mathrm{c}^{-1} \text {; }
$$

Следовательно:

$$
\begin{gathered}
\omega_{1}=\sqrt{\frac{\mathrm{K}_{1}}{\mathrm{~m}}}=\sqrt{\frac{31600}{945}}=18,2 ; \\
\mathrm{n}_{1}=\frac{12400}{2 \cdot 945}=6,55 ; \\
\omega_{1}{ }^{\prime}=\sqrt{\omega_{1}^{2}-\mathrm{n}_{1}^{2}}=16,7 .
\end{gathered}
$$

Примем продолжительность первой фазы равной 0,06 с. Тогда

$$
\Delta=\frac{\mathrm{V}_{0}}{16,7} \cdot \mathrm{e}^{-6,55 \cdot 0,06} \cdot \sin (16,7 \cdot 0,06)=0,34 \cdot \mathrm{V}_{0} .
$$

Увеличим значение $\tau_{1}$ на $33 \%$, т.е. на 0,08 с. Тогда

$$
\Delta=\frac{\mathrm{V}_{0}}{16,7} \cdot \mathrm{e}^{-6,55 \cdot 0.08} \cdot \sin (16,7 \cdot 0,08)=0,0344 \cdot \mathrm{V}_{0} .
$$

Уменьшая значение $\tau_{1}$ до 0,05 с, т.е. на $20 \%$. Тогда

$$
\Delta=\frac{\mathrm{V}_{0}}{16,7} \cdot \mathrm{e}^{-6,55 \cdot 0,05} \cdot \sin (16,7 \cdot 0,05)=0,032 \cdot \mathrm{V}_{0} .
$$

Таким образом, если учитывать изменение $\tau_{1}$ с изменением начальной скорости, то это, по-видимому, не должно внести существенных корректив и в рассматриваемом диапазоне зависимость $\Delta=\Delta\left(\mathrm{V}_{0}\right)$, в первом приближении можно считать линейной:

$$
\Delta=\mathrm{X} \cdot \mathrm{V}_{0} \text {. }
$$

На основании изложенного представляется возможным рекомендовать следующий метод прогнозирования безопасной скорости автомобиля перед его испытаниями на столкновение. Основой метода является экспериментально-расчетный график, примерный вид которого показан на рис.1. В левой части графика наносятся функциональные зависимости между перемещением травмоопасных деталей $(\delta)$ и максимальной деформацией передней части (S). На рис.1 кривая 1 иллюстрирует перемещение панели приборов. Точки указанных кривых получены экспериментальным путем при испытаниях на удар автомобиля ВАЗ-2108. В правой части графика нанесена прямая 2, рассчитанная в соответствии с формулой (45) для того же автомобиля. При этом приняты следующие исходные данные: $\mathrm{m}=945 \kappa г, \mathrm{~V}_{0}=14 \mathrm{M} / \mathrm{c}, \omega_{1}^{\prime}=16,7, \tau=0,05 \mathrm{c}$.

Перед испытаниями в результате обмера внутреннего интерьера салона и определения параметров жизненного пространства устанавливают максимально допустимое перемещение травмоопасных деталей $\delta_{\max }$ из условий сохранения пассивной безопасности. Отложив это значение на шкале $\delta_{\mathrm{i}}$, проводим вертикаль до кривой, соответствующей данной детали. Из точки пересечения ведут горизонталь до встречи с прямой в правой части графика. Абсцисса точки пересечения укажет максимально допустимую скорость автомобиля. 


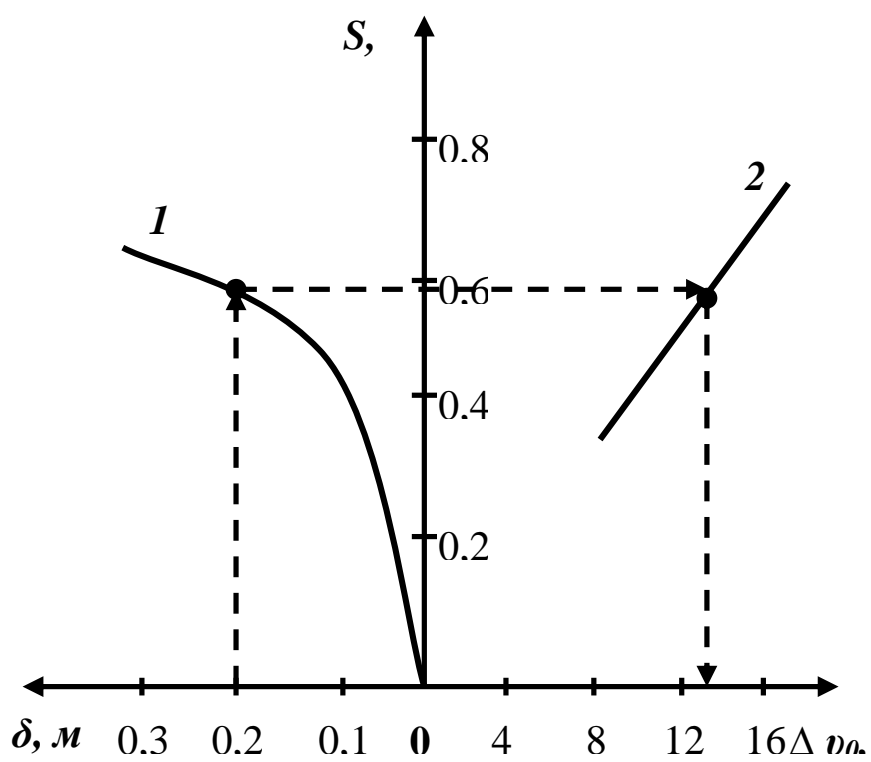

Рисунок 1 - Экспериментально-аналитический метод прогнозирования начальной скорости автомобиля ВАЗ-2108

На графике (см. рис.1) это точка А, т.е. значение максимально допустимой скорости столкновения для данной модели автомобиля можно считать приблизительно $\mathrm{V}_{\mathrm{a}}=60 \mathrm{\kappa м} / \mathrm{s}$.

C учетом использования данных экспериментов (краш-тестов), проведенных на Автополигоне авторами, получены также зависимости деформации автомобиля от $\Delta \mathrm{V}$ :

- для автомобилей ВАЗ-2108 (09):

$$
\mathrm{V}_{\mathrm{a}}=21.9 \cdot \mathrm{S}_{\mathrm{a}}^{0.42}
$$

- для автомобилей ГАЗ-3102, 3110:

$$
\mathrm{V}_{\mathrm{a}}=27,4 \cdot \mathrm{S}_{\mathrm{a}}^{0.6}
$$

Полученные зависимости могут быть использованы при проведении автотехнических экспертиз. Путем измерения обычной линейкой величины остаточной деформации или перемещение панели приборов в салоне автомобиля, полученные формулы позволяют с достаточной точностью определить начальную скорость автомобиля.

\section{Библиографический список:}

1. Рябчинский А.И., Иларионов В.А., Пчелин И.К., Кузнецов О.Г. Моделирование процесса наезда автомобиля на неподвижное препятствие. Сб. научных трудов НАМИ «Полигонные испытания, исследование и совершенствование автомобилей» -М.: 1987.c. $45-46$.

2. Илларионов В.А. Экспертиза дорожно-транспортных происшествий. -М.: Транспорт, 1989.-254c.

3. Батманов Э.З. Усовершенствование системы сбора и анализа данных ДТП. Московский автомобильно-дорожный институт (государственный технический университет). - М.,2003г.-13с. Деп. в ВИНИТИ 30.10.2003г., № 1890-В2003. 